\title{
Effect of the Cation Size and of the Solvent Composition on the Conformation of Poly(L-glutamic acid) Alkaline Metal Salts
}

\author{
Hubert DAOUST and Daniel ST-CYR \\ Department of Chemistry, Université de Montréal, \\ P. O. Box 6210, Station A, Montreal, Quebec, Canada, H3C $3 \mathrm{VI}$
}

(Received October 28, 1981)

\begin{abstract}
The coil $\rightarrow$ helix transition in aqueous mixed solvents of alkaline metal salts of poly(L-glutamic acid) has been studied by circular dichroism (CD) as a function of solvent composition and time. Four organic solvents were used, dioxane, 2-chloroethanol (CE), 2,2,2trifluoroethanol (TFE), and tetrahydrofuran (THF). CD spectra were taken just after the dissolution of the polypeptide, after three days and after seven days. In the water-dioxane pair, the transition is almost complete at the solubility limit of $\mathrm{Li}^{+}$and $\mathrm{Na}^{+}$salts $(\sim 70 \%$ by weight of dioxane); for the $\mathrm{K}^{+}, \mathrm{Rb}^{+}$, and $\mathrm{Cs}^{+}$salts, the helicity does not go over $20 \%$ even after seven days. When CE is used as organic solvent, the transition appears at a rather low CE content $(25-35 \%)$ and is complete for all the salts. The transition occurs also for the salts up to their solubility limit in water-TFE but at high TFE content $(\sim 80 \%)$. No transition takes place when THF is used as the organic solvent. A time dependent phenomenon in the helical content was found with dioxane and CE as organic solvents but none with TFE. The results are discussed in the light of the work of Morcellet and Loucheux.

KEY WORDS Poly(L-glutamic acid) Salts / Solvent Induced Conformational Changes / Coil to Helix Transition / Water Soluble Polypeptides / Helicity /
\end{abstract}

It is well known that a fraction of counterions tend to condense on polyelectrolyte chains even at very high dilution. ${ }^{1}$ This fraction can be estimated to $1 / \lambda$ where $\lambda(>1)$ is the charge density parameter. $^{2}$ In the case of alkaline metals polycarboxylates, it has been shown that ionic association between polyions and counterions is more pronounced for small cations than for large cations. ${ }^{3}$ This ionic condensation or association plays an important role in thermodynamic properties of strong polyelectrolyte solutions and also in the conformation of the chains. Furthermore, the ionic condensation depends on the nature of the solvent and in the case of aqueous mixed solvents, it depends on the content of the organic solvent.

Many investigators have studied the effect of many agents on the coil $\rightarrow$ helix transition of poly(Lglutamic acid) (PLGA). For instance, Iizuka and Yang ${ }^{4}$ have studied the effect of added salts and of different solvents on the $\mathrm{pH}$-induced transition of
PLGA. Others ${ }^{5-7}$ have investigated the effect of the composition of mixed solvents at constant $\mathrm{pH}$. This last type of investigation gives results which depend on the degree of neutralization of PLGA, i.e., on the selected value for $\mathrm{pH}$.

It seemed of interest to make a systematic study of the effects of both the cation size and the composition of aqueous mixed solvents on the coil $\rightarrow$ helix transition of alkaline metal salts of PLGA. The investigation was done at complete neutralization for each salt and in absence of added salts. Thus, the only factors affecting the conformational transition are the size of the cation, the nature of the organic solvent and the composition of the aqueous mixed solvent. The selected organic solvents are dioxane, 2-chloroethanol (CE), 2,2,2-trifluoroethanol (TFE), and tetrahydrofuran (THF). Moreover, a time dependent phenomena in the transition region, i.e., an increase in the helicity of ionizable polypeptides with time has been already re- 
ported. ${ }^{8-10}$ The same effect was noticed in some of the systems investigated in the present work and a mechanism for this effect is discussed.

\section{EXPERIMENTAL}

\section{Materials}

The PLGA sample was prepared by debenzylation of $\operatorname{poly}(\gamma$-benzyl-L-glutamate) (PBLG) by $\mathrm{HBr}$ in benzene according to a method described by Idelson and Blout. ${ }^{11}$ The PBLG sample was prepared according to a method described by Blout and Karlson $^{12}$ and its viscosity molecular weight was 240,000 based on calibration curves of Doty et al. ${ }^{13}$ The weight average degree of polymerization of the resulting PLGA was 390 as determined by sedimentation in $\mathrm{NaCl} 1 \mathrm{M}$ according to the synthetic boundary technique of Klainer and Kegeles. ${ }^{14}$

All alkaline metal salts were prepared by neutralization of PLGA by an excess of the corresponding hydroxide. The resulting solutions were dialyzed against deionized distilled water down to a $\mathrm{pH}$ corresponding to the equivalence point $(\sim 8.2-$ 8.7). The salts were then recovered by freeze drying; potentiometric analysis showed no presence of acid residue in any of the salts. Water content of the dried salts was determined by thermal gravimetric analysis.

A survey of the literature showed that many investigators ${ }^{4,5,10,15}$ have prepared PLGA alkaline metal salts in a very similar way except that in most cases the resulting salt solutions were dialyzed down to a $\mathrm{pH}$ between 7 and 8 corresponding to the degree of neutralization between 90 and $97 \%$.

Stock solutions were prepared by dissolving salts in water first $(\mathrm{pH} \sim 8.3)$ and then by adding the organic solvent. Dissolving a dried salt directly in water-dioxane or water-TFE pairs freezes the chains in such structures so that no transition occurs. All organic solvents were of reagent grade and distilled under argon before use.

\section{Circular Dichroism Measurements}

CD measurements were made with a Cary 61 spectropolarimeter at $22^{\circ} \mathrm{C}\left( \pm 1^{\circ}\right)$. A quartz cell (Hellma) with an optical path length of $10^{-4} \mathrm{~m}$ was used. During the measurements, the optical path of the instrument was purged with nitrogen at $1.1 \times 10^{-2} \mathrm{~m}^{3} \mathrm{~min}^{-1}$. The spectropolarimeter was calibrated with $d$-10-camphorsulfonic acid accord- ing to the method described by DeTar. ${ }^{16}$ The mean residue ellipticity at $222 \mathrm{~nm},[\Theta]_{222}$ in $\operatorname{deg} \mathrm{cm}^{2}$ $\mathrm{dmol}^{-1}$ was calculated according to the relationship

$$
[\Theta]_{222}=\frac{\Theta_{222} M_{0}}{10 l C}
$$

where $\Theta_{222}$ is the observed ellipticity in degrees at $222 \mathrm{~nm}, M_{0}$ is the molecular weight of the residue, $l$ is the pathlength of the cell in $\mathrm{cm}$, and $C$, the concentration in $\mathrm{g} \mathrm{cm}^{-3}$. Solutions used for the measurements were prepared from stock solutions; their concentration was close to $0.125 \%$ by weight. During the time dependence study, solutions were kept at room temperature to prevent any temperature effect on the results.

\section{RESULTS AND DISCUSSION}

\section{Water-Dioxane}

This is one of the most commonly used aqueous mixed solvents in conformational studies of watersoluble polypeptides. In Figure 1, the value of $[\Theta]_{222}$ is plotted as a function of the composition of the mixed solvent for each salt on the seventh day following dissolution. The coil $\rightarrow$ helix transition is complete in the case of $\mathrm{Li}^{+}$and $\mathrm{Na}^{+}$salts, the midpoint of the transition corresponding to 72 and $63 \%$ by weight of dioxane. The behavior of the three other salts, $\mathrm{K}^{+}, \mathrm{Rb}^{+}$, and $\mathrm{Cs}^{+}$is about the same but in contrast to the first two small cation salts, the transition is never completed. It should be mentioned that the last point of each curve in Figure 1 represents the solubility limit of the salt.

According to Morcellet and Loucheux, ${ }^{17}$ there is a preferential solvation of PLGNa by dioxane far from the transition but just before the conformational change, water becomes preferentially bound to the coil and then partial dehydration occurs when the transition is induced by further addition of dioxane. The lowering of the dielectric constant caused by the presence of dioxane in the mixed aqueous solvent favors cation association with carboxylate groups and the helix formation is allowed due to the decrease in repulsion between negative sites on side groups along the chain. Since $\mathrm{Li}^{+}$and $\mathrm{Na}^{+}$associate strongly with polycarboxylates, the coil $\rightarrow$ helix transition occurs easily. For the three remaining cations, the conformational change is only partial in spite of the low dielectric constant $(\sim 12)$ at $80 \%$ of dioxane ${ }^{18}$ since 


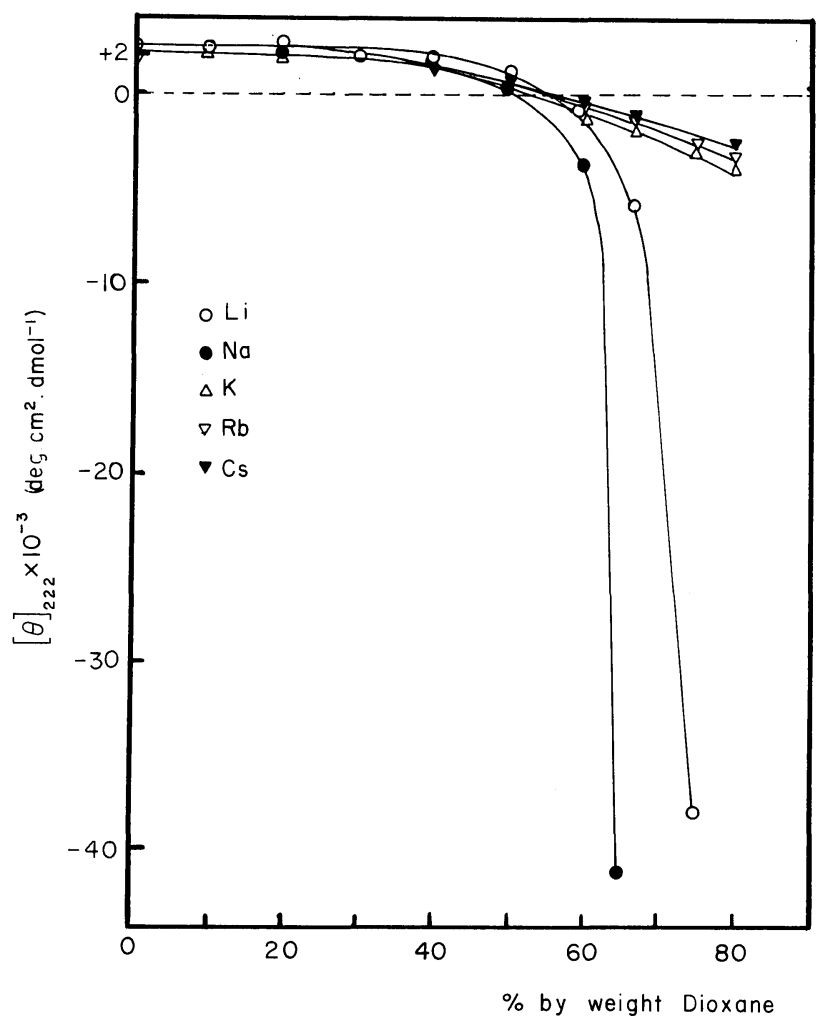

Figure 1. Variation in molar ellipticity for PLGA salts as a function of dioxane content on the seventh day.

the degree of ionic association of these cations with carboxylate groups is smaller than in the case of the first two.

\section{Water-CE}

This aqueous mixed solvent has been used recently by many authors. ${ }^{5,19}$ Figure 2 shows that in this solvent, transition occurs at a low organic constituent content (between 20 and $30 \%$ by weight) for all the salts and seems to be complete excepted that $[\Theta]_{222}$ is noticeably larger (in absolute value) for $\mathrm{Cs}^{+}$than for the other four cations. This is probably a residue molar weight effect. The lowering of the dielectric constant is not the major factor inducing the transition in this solvent since its value is close to 64 at $30 \%$ by weight of CE. ${ }^{5}$ In this case, the coil $\rightarrow$ helix transition is rather of the $\mathrm{pH}$-induced type as proposed by Morcellet and Loucheux ${ }^{5}$ who found that CE preferentially binds to carboxylate groups. According to these authors, this is probably due to the strong attraction of the labile $\mathrm{H}$ atom of the $\mathrm{OH}$ group of $\mathrm{CE}$ by the carboxylate group; the concentration of a positive charge on the $\mathrm{H}$ atom is obviously due to the deshielding effect of the chlorine atom on $\alpha$-carbon. The strong association between $\mathrm{CE}$ molecules and carboxylate groups lowers the electrostatic repulsion between negative sites along the chain and the coil $\rightarrow$ helix transition becomes possible. This mechanism would also explain the inversion of the order in which the transition takes place for different salts as compared to that observed in water-dioxane.

\section{Water-TFE}

This mixed solvent is often used in conformational studies of proteins. ${ }^{20}$ As shown in Figure 3 , all the investigated salts undergo the coil $\rightarrow$ helix transition but at rather high and almost the same TFE content. In order to explain these results, one may refer to the two mechanisms already proposed for the first two aqueous mixed solvents. The $\mathrm{Li}^{+}$ and $\mathrm{Na}^{+}$salts go to a complete transition before 


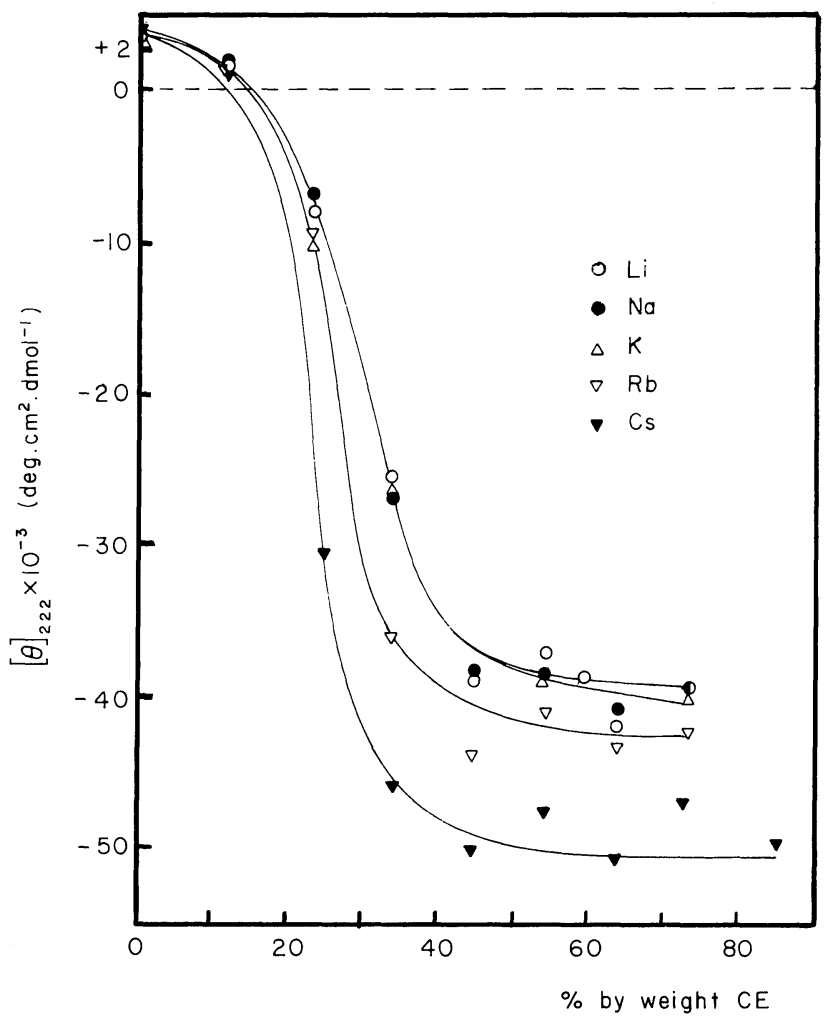

Figure 2. Variation in molar ellipticity for PLGA salts as a function of CE content on the seventh day.

they become insoluble and the $\mathrm{Cs}^{+}, \mathrm{Rb}^{+}$, and $\mathrm{K}^{+}$ salts reach the solubility limit with only a partial transition in the given decreasing order. If the dielectric constant were the only factor responsible for the transition, the order for the larger cations would have been reversed. If, on the contrary, the preferential solvation of TFE on carboxylate groups by itself could explain the transition, it would have been more complete for $\mathrm{K}^{+}, \mathrm{Rb}^{+}$, and $\mathrm{Cs}^{+}$than for $\mathrm{Li}^{+}$and $\mathrm{Na}^{+}$salts. However, this last mechanism can explain only partly the results obtained for the large cations salts. Since the deshielding effect of fluorine atom on $\alpha$-carbon is weaker than that of chlorine atom, it takes more TFE than $\mathrm{CE}$ to induce the transition. The dielectric constant effect should be discarded to explain the transition of the last three salts since its value is 37 at $85 \%$ by weight of $\mathrm{TFE}^{21}$ whereas only 9 for the same dioxane content ${ }^{18}$ and no transition is then possible for these salts as discussed above.

For the $\mathrm{Li}^{+}$and $\mathrm{Na}^{+}$salts, the conformational transition can be explained by a combination of both the dielectric constant effect and the preferential solvation effect. Since these two small cations condense more easily on negative sites, a large amount of these are neutralized by cations and the rest of the sites are solvated by TFE inducing finally the transition. This could also be considered as a $\mathrm{pH}$-induced transition.

\section{Water-THF}

THF has been utilized because of its similitude with dioxane but it has a higher dielectric constant, $7.35^{22}$ as compared to $2.21 .^{23}$ However, no transition takes place in this mixed solvent even at $85 \%$ by weight of THF. Only a monotonic decrease in $[\Theta]_{222}$ was noticed but its value was always positive in spite of the low value of the dielectric constant for the highest content of THF $\left(\sim 17^{21}\right)$. It is known that dioxane has some interaction with the peptide bond $^{5,17}$ and THF being much more polar, may react more strongly with that bond through the formation of $\mathrm{H}$-bonding between $\mathrm{NH}$ groups and furanosic oxygen. This is the type of interaction for 


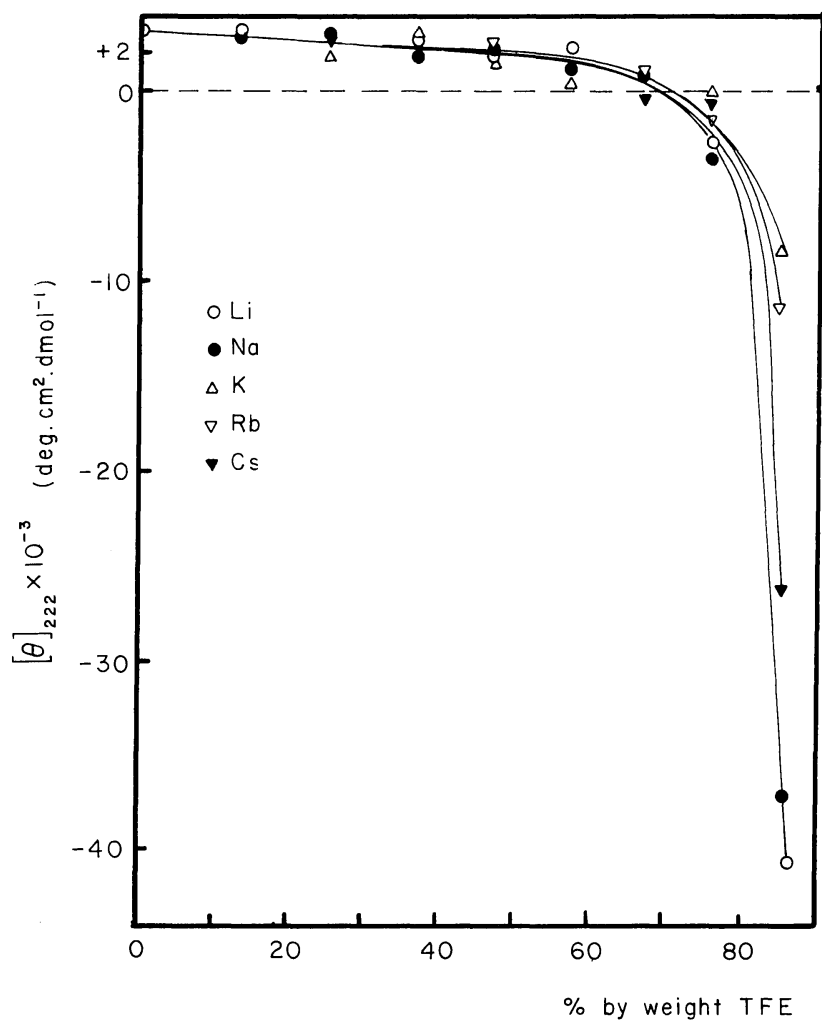

Figure 3. Variation in molar ellipticity for PLGA salts as a function of TFE content on the seventh day.

most of the organic solvents which induce the helix $\rightarrow$ coil transition of non-ionic polypeptides.

\section{Time Dependence of the Coil $\rightarrow$ Helix Transition}

It was found in many cases studied here that ellipticity in the transition region of a given salt increses with time for a period up to seven days following dissolution. After this, no variation in $[\Theta]_{222}$ was noted for any of the salts. As mentioned above, a similar effect was observed for PLGNa ${ }^{8,10}$ and for poly $(\mathrm{L}-\mathrm{lysine}, \mathrm{HBr})^{9}$ in aqueous mixed solvents.

The observed time dependent effect has been partly explained by Morcellet and Loucheux ${ }^{10}$ as being a diffusion phenomenon induced by the difference in solvent composition between the solvation layer and bulk. This explanation is used as the starting point in the following discussion. But before going any further, it should be recalled that the solvent induced coil $\rightarrow$ helix transition in the case of ionizable polypeptides is accompanied by partial dehydration of the macromolecule. ${ }^{5,24}$

This time dependence of $[\Theta]_{222}$ is negligible for all the salts in the water-TFE system where transition appears at rather high content of TFE. However, this time dependence is very important when $C E$ is used as the organic solvent as shown in Figure 4 for PLGLi. The other four salts show similar behavior to PLGLi in this mixed solvent. The effect of cation size is underlined in Figure 5 where $\Delta[\Theta]_{222}$ i.e., the difference between the values of $[\Theta]_{222}$ obtained on the seventh day and following dissolution of the salts, is expressed as a function of CE content. This figure was obtained from figures similar to Figure 4. $\Delta[\Theta]_{222}$ is a representation of the amplitude of change in the helix content for a given period of time and should not be interpreted as the rate of dehydration of the polymeric coil. A low value for $\Delta[\Theta]_{222}$ indicates that for a given amount of organic solvent, no significant change in the helix content of the polymer occurs over a period of seven days. A large value for $\Delta[\Theta]_{222}$ 


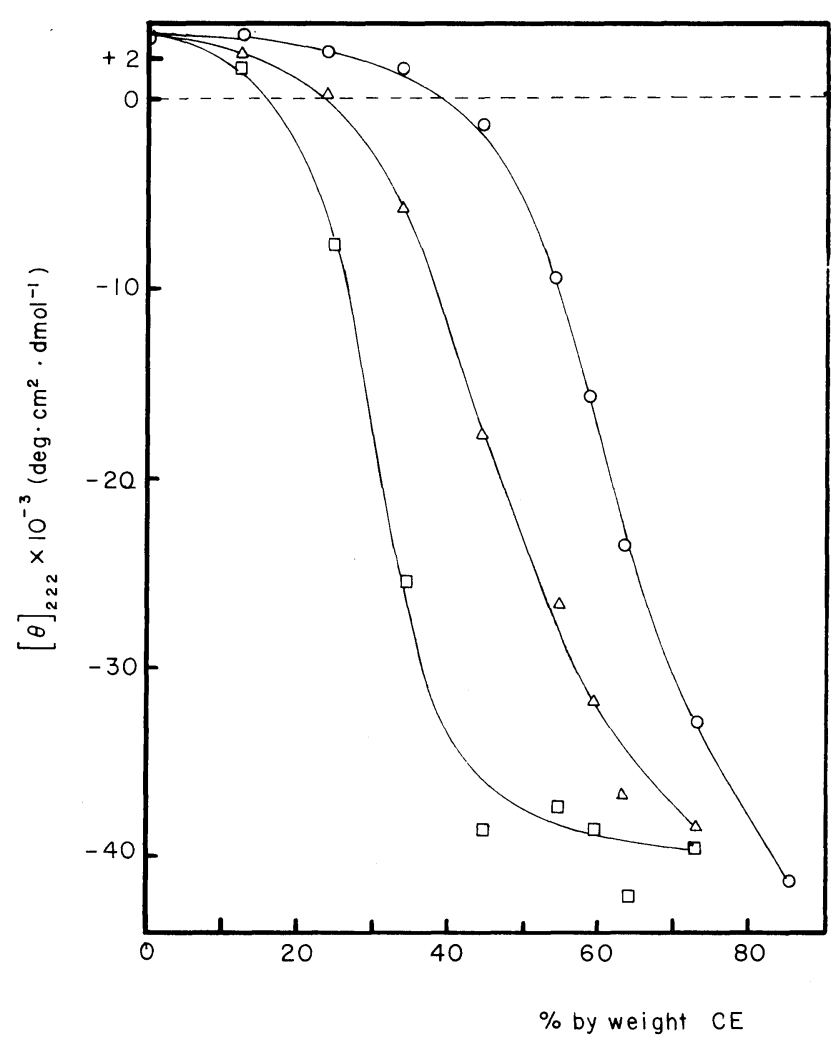

Figure 4. Variation in molar ellipticity for PLGLi in water-CE system: $\bigcirc$, just after dissolution; $\triangle$, on the third day; $\square$, on the seventh day following dissolution.

means that the helix content has increased significantly with time, the percentage of the organic solvent remaining the same. The results show that the maximum of the curve appears at decreasing CE content and becomes more important as the cation size increases. This effect of cation size on $\Delta[\Theta]_{222}$ is also important in water-dioxane pair for $\mathrm{Li}^{+}$and $\mathrm{Na}^{+}$for which the highest value for $\Delta[\Theta]_{222}$ corresponds to the solubility limit.

The rate of dehydration of the solvation layer depends on the difference in water activity between this layer and the bulk. Of course, this difference is a function of the organic solvent content in the conformational transition region. The rate of interdiffusion of both water molecules and organic solvent molecules between the solvation layer and the bulk is then quite large for the water-TFE pair but is much smaller for that of water-CE.

In the case of the water-dioxane pair, the time dependent effect is still very important but occurs on a narrower spread of the organic solvent content as shown in Figure 5. The coil $\rightarrow$ helix transition being induced at a higher content of the organic solvent, the rate of the dehydration phenomenon is larger than for CE. The effect of cation size is still easily noticeable in this case.

In conclusion, the effect of cation size on the solvent induced coil $\rightarrow$ helix transition of poly(Lglutamic acid) salts underlines the role of the dielectric constant of aqueous mixed solvents on this conformational change. But for a $\mathrm{pH}$-induced type transition, the effect of cation size becomes much less important since counterion condensation is not the major factor in this case. The mechanism of the time dependent phenomenon accompanying the conformational change as proposed by Morcellet and Loucheux is acceptable but the content of the organic solvent at which this change occurs must be taken into consideration. 


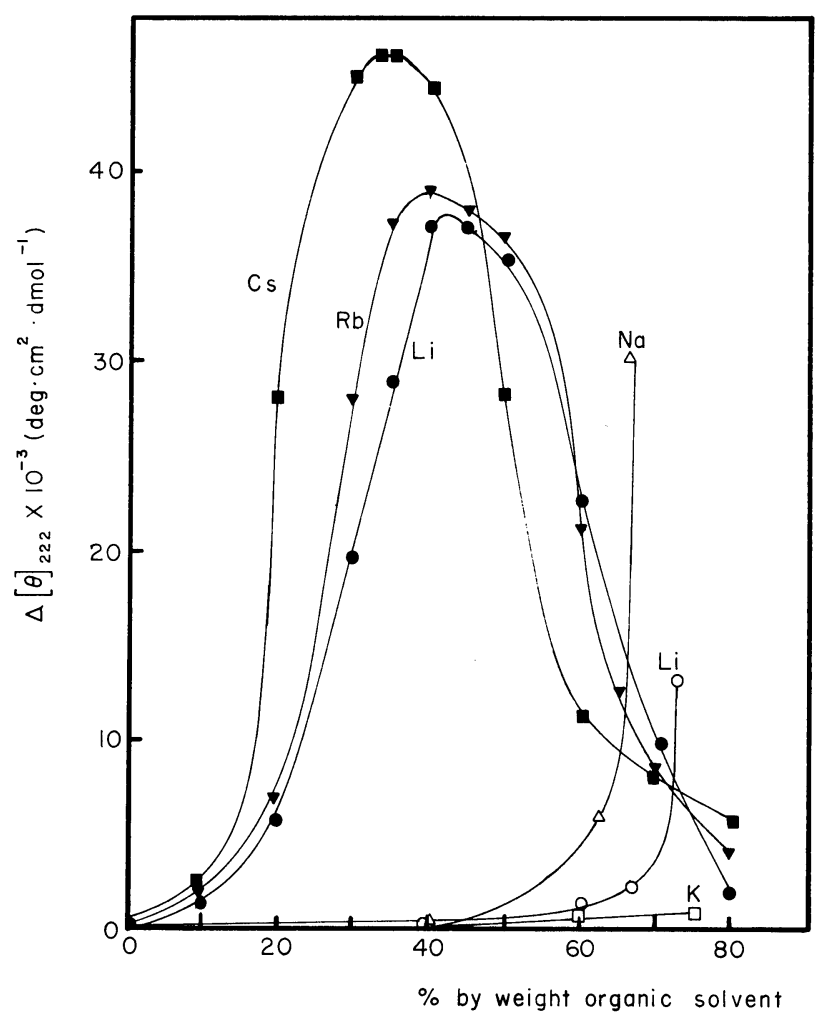

Figure 5. Variation in the difference between the molar ellipticity on the seventh day and following dissolution as a function of organic solvent content: filled points, water-CE pairs; open points, waterdioxane.

Acknowledgements. Thanks are due to Dr. Patrick Schiller of the Institut de Recherches Cliniques de Montréal for use of the Carey. spectropolarimeter. The Natural Sciences and Engineering Research Council of Canada and the Department of Education of Quebec are acknowledged for their financial support.

\section{REFERENCES}

1. M. Nagasawa, "Polyelectrolytes," Vol. 1, E. Sélégny, Ed., D. Reidel Publishing Co., Dordrecht, Boston, 1974.

2. G. A. Manning, J. Chem. Phys., 51, 924 (1969).

3. H. Daoust and M.-A. Chabot, Macromolecules, 13, 616 (1980).

4. E. Iizuka and J. T. Yang, Biochemistry, 4, 1249 (1965)

5. M. Morcellet and C. Loucheux, Biopolymers, 19, 2177 (1980).

6. P. Doty, A. Wada, J. T. Yang, and E. R. Blout, J.
Polym. Sci., 23, 851 (1957).

7. N. E. Bychkova, O. B. Ptitzyn, and J. V. Barskaya, Biopolymers, 10, 2161 (1971).

8. M. L. Tiffany and S. Krimm, Biopolymers, 6, 1379 (1968).

9. M. Barteri and B. Pispisa, Biopolymers, 12, 2309 (1973).

10. M. Morcellet and C. Loucheux, Eur. Polym. J., 14, 697 (1978).

11. M. Idelson and E. R. Blout, J. Am. Chem. Soc., 80, 4631 (1958).

12. E. R. Blout and R. H. Karlson, J. Am. Chem. Soc., 78, 941 (1956).

13. P. Doty, J. H. Bradbury, and A. M. Holtzer, J. Am. Chem. Soc., 78, 747 (1956).

14. S. M. Klainer and G. Kegeles, J. Phys. Chem., 59, 952 (1955).

15. D. A. Brandt and P. J. Flory, J. Am. Chem. Soc., 87, 2788 (1965).

16. D. F. DeTar, Anal. Chem., 41, 1406 (1969).

17. M. Morcellet and C. Loucheux, Polymer, 16, 401 (1975).

18. J. Timmermans, "The Physico Chemical Constants 


\section{H. Daoust and D. ST-CyR}

of Binary Systems in Concentrated Solutions," Vol. 4, Interscience Publishers Inc., New York, 1960.

19. S. N. Timasheff, Acc. Chem. Res., 3, 62 (1970).

20. E. Nabedryk-Viala, C. Thierry, P. Calvet, S. Fermandjian, L. Kisfaludy, and J. M. Thierry, Biochem. Biophys. Acta, 536, 252 (1978).

21. Unpublished data.
22. V. Sedivec and J. Flek, "Handbook of Analysis of Organic Solvents," John Wiley and Son, New York, 1976.

23. A. A. Maryott and E. R. Smith, N. B. S. Circular 514, Washington, D. C.

24. T. Mori, J. Komiyama, and T. Iijima, J. Chem. Soc. Faraday Trans. 1, 74, 2583 (1977). 